\title{
Scale-up and Modeling of Oxygen Diffusion Electrodes for Chlorine-Alkali Electrolysis II. Effects of the Structural Parameters on the Electrode Performance Based on the Thin-Film and Flooded-Agglomerate Model
}

\author{
Xiao-Lin WANG ${ }^{\dagger}$ and Seiichiro KODA*
}

Received June 3, 1997 ; Accepted August 11, 1997

The performance of the oxygen diffusion electrode in a chlorine-alkali electrolysis cell system, which is expressed by the curve of the overpotential vs. the current density, has been investigated based on the TFFA (Thin-Film and Flooded-Agglomerate) model as functions of various structural parameters of the electrode. The electrode consists of the reaction layer and gas-diffusion layer. The reaction layer is assumed to be composed of cylindrical porous agglomerates and surrounding hydrophobic channels. Among the investigated structural parameters, the porosity of the reaction layer, the thickness of the thin-film on the flooded-agglomerates, the radius of the floodedagglomerates, and the volume fraction of the flooded-agglomerates in the reaction layer are found to considerably affect the electrode performance. The radius of the gas channels in the reaction layer and the porosity and average pore radius of the gasdiffusion layer are less important. It is because that the Knudsen diffusion of oxygen is much faster than the transport and reaction of dissolved oxygen in the thin-film and flooded-agglomerates of the reaction layer of the electrode.

\section{INTRODUCTION}

Polytetrafluoroethylene(PTFE)-bonded carbon oxygen diffusion electrodes received considerable attention because they offer a high surface area and high electrocatalytic activity for current generation in fuel cell systems. ${ }^{1.6)}$ However, the industrial applications of oxygen diffusion electrodes, such as to replace the conventional hydrogen evolution cathodes in chlorine-alkali membrane cell electrolyzers, are not realized

Department of Chemical System Engineering, School of Engineering, The University of Tokyo (Hongo 7-3-1, Bunkyo-ku, Tokyo 113, Japan)

$\dagger$ Present address: Department of Polymer Engineering, Nanjing University of Chemical Technology (Nanjing 210009, P. R. China)

Key Words: Chlorine-alkali electrolysis, Oxygen diffusion electrode, Thin-film and flooded-agglomerate model, Electrode performance so far because of many difficulties in the realization of scale-up and structure optimization of the oxygen cathodes. ${ }^{78)}$

In the preceding paper, $\left.{ }^{8}\right)$ the available range of the pressures of the catholyte chamber and gas-supply chamber was obtained from the analysis of the hydrostatic force balance to prevent from gas percolation into the catholyte chamber and catholyte flooding into the gassupply chamber. The position of the gas-liquid interface was changeable with the pressure difference between the catholyte chamber and the gas-supply chamber, the depth of catholyte, and the distributions of the contact angle of the reaction layer and gas-diffusion layer of the cathode. The degradation of electrode performance was also predicted for the case that the gas-liquid interface stayed at the gasdiffusion layer. However, based on a simplepore-and-meniscus model, the limiting current density of the cathode was estimated simply as a function of the two parameters, i.e., the pore 
radius and the porosity of the reaction layer, and was not adequate to be used as a measure for the optimization of the cathode structure. Further detailed mathematical analysis and simulation of the electrode performance are necessary to support the optimization of the electrode structure or the improvement of the preparation methods in the scale-up of an oxygen diffusion electrode used in a chlorinealkali electrolysis system.

In the past 30 years, several mathematical models have been proposed to describe the behaviors of gas diffusion electrodes in terms of the properties of the electrode structure. The simplest model is the simple-pore-andmeniscus model. ${ }^{9 \cdot 13)}$ This model assumes that reactant gas diffuses into the pores of a porous electrode partly filled with electrolyte, dissolves at the gas-electrolyte meniscus interface and further diffuses in solution to reach the catalytic sites. So the simple-poreand-meniscus model is quite primitive, and mostly underestimates a value for the diffusion-limiting current density, since most of the current is concentrated in a small part of the pore walls of the electrode. The thin-film model ${ }^{9,14-18)}$ is an extension of the simple-poreand-meniscus model and accounts for variations in the wetting tendency of the electrolyte on the pore wall in excess pressure of gas phase. The electrode reaction, although less restricted, still tends to be concentrated in the part of the film which is close to the bulk catholyte. Since real porous electrodes have a spectrum of pore sizes, the floodedagglomerate model was proposed.18 ${ }^{22)}$ This model assumes the reaction layer of the electrode with a double-scale of porous structure, in which catalyst particles are assumed to form cylindrical porous agglomerates flooded with electrolyte, and the surrounding hydrophobic channels are filled with reactant gas. The reactant gas diffuses in the radial direction of the cylindrical floodedagglomerate, while ionic current is conducted in the axial direction. This approach involves a more effective utilization of the electrode as compared to the thin-film model and the simple-pore-and-meniscus model. However, there is no film on the external surface of the flooded-agglomerates, and the concentration polarization could not be considered. By taking into consideration of those shortcomings, Cultip and coworkers ${ }^{23,}$ 24) extended a new model from the flooded-agglomerate model and used it to describe the transport and reaction processes of a polytetrafluoroethylene (PTFE) bonded platinum-on-carbon oxygen diffusion electrode in a fuel cell. The Cultip's model was called a thin-film flooded-agglomerate (TFFA) model and applied later to estimate the performance and structure properties of various kinds of gas diffusion electrodes. ${ }^{25} \cdot 40$ )

So far, very few works have been focused on optimization of the electrode structure from the simulation results of the electrode performance. Many experimental studies ${ }^{1-6,30}$. 35) suggested that the TFFA model represents the structure of an oxygen diffusion electrode more adequately than the other models. Therefore, we will use the TFFA model to give a detailed description of all processes that include diffusion of oxygen in the gas-diffusion layer and the gas-channels of the reaction layer, dissolution and diffusion of oxygen in the thinfilm of the reaction layer, diffusion and reaction of oxygen in the flooded-agglomerates of the reaction layer, and the ionic and electronic conduction. Then we will select and determine appropriately a set of values for the base-case conditions of the structural parameters of the oxygen diffusion electrode to be scaled up. Lastly, we will estimate the effects of the structure parameters on the electrode performance which is represented by the curve of overpotential vs. current density, and determine which structural parameter is important to affect the electrode performance.

\section{THEORETICAL}

\subsection{Assumptions of the TFFA model}

The oxygen diffusion electrode serves as a cathode in a $\mathrm{NaCl}$ electrolysis system. As shown in Fig. 1, the oxygen diffusion electrode possesses a double layered structure. The gas diffusion layer is made from the hydrophobic 


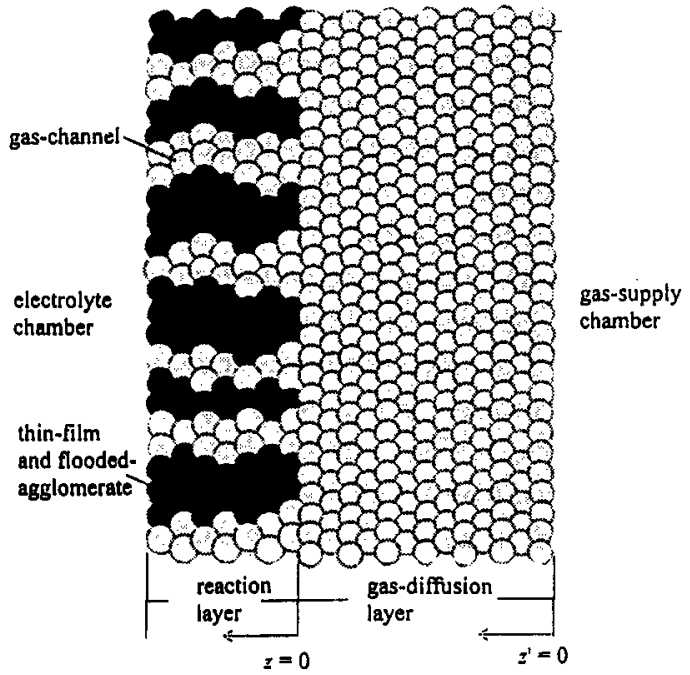

Fig. 1 Model of structure of gas-diffusion electrode.

activated carbon powder and PTFE dispersion, and the reaction layer is prepared from the platinum catalyst particles, the hydrophilic and hydrophobic activated carbon powder and PTFE dispersion..$^{1-6)}$ The following assumptions are made according to refs. 23-29.

(1) The gas diffusion layer contains only the gas filled pores expressed with the structural parameters of an average pore radius, $r_{B}$, uniform porosity, $\varepsilon_{\mathrm{B}}$, and tortuosity, $\tau_{\mathrm{s}}$.

(2) The reaction layer has a distribution of pore sizes with uniform porosity and tortuosity. It is generally accepted that with the increase of pore radius, the wettability of pore walls changes from hydrophilic to hydrophobic.

(3) Large pores are filled with gases and called gas-channels. The average radius, free volume fraction and tortuosity of gas-channels are denoted as $n, a$, and $\tau$, respectively.

(4) Small pores are flooded with the electrolyte and called flooded-agglomerates. The flooded-agglomerates are regarded as cylindrical columns with an average radius, $r_{a g}$; and its volume fraction against all of the reaction layer volume is denoted as $\chi_{a g}$.

(5) There is a thin-film of electrolyte with the thickness, $\delta$ h, between the floodedagglomerates and the gas-channels. The area of gas-electrolyte interface is equal to that of the thin-films denoted by $S_{\text {If }}$.

(6) Since the radii of the pores of gas diffusion layer and gas-channels of reaction layer are smaller than $90 \mathrm{~nm}$, Knudsen diffusion can be considered as a dominant term of oxygen diffusion in the present work.

(7) Dissolution of oxygen into the electrolyte is fast, so that the Henry's law can be applied to express the relation of the partial pressure of oxygen in the gas-channel and the concentration of dissolved oxygen in the electrolyte.

(8) The Fick's law is useful to represent the diffusion of dissolved oxygen across the thinfilm of electrolyte to flooded-agglomerates.

(9) The reaction of oxygen in floodedagglomerates is expressed by a direct $4 \theta^{-}$ reduction process. ${ }^{41)}$

$$
\mathrm{O}_{2}+2 \mathrm{H}_{2} \mathrm{O}+4 \theta^{\circ} \rightarrow 4 \mathrm{OH} \cdot(+0.401 \mathrm{~V})
$$

The transport and reaction of dissolved oxygen in flooded-agglomerates are treated according to a general case of the diffusion with a first-order reaction of oxygen concentration.

\subsection{Basic equations of the TFFA model}

2.2.1 Oxygen diffusion in gas diffusion layer

The diffusion of oxygen in a porous medium is usually expressed by the StefanMaxwell equations. 23, 24) According to assumption (6), the diffusion of oxygen through the gas diffusion layer can be expressed by the following equation.

$$
\frac{d P_{O_{2}}}{d z^{\prime}}=-\frac{R T J_{O_{2}}}{D_{k s} \varepsilon_{s} / \tau_{s}}
$$

Here, $\boldsymbol{H}_{2}$ and $J_{2}$ are the partial pressure and flux of oxygen; $\varepsilon_{\mathrm{s}}$ and $\tau_{\mathrm{s}}$ are the porosity and tortuosity of the gas diffusion layer; $D_{\mathrm{ks}}$ is the Knudsen diffusion coefficient of oxygen in the gas-diffusion layer and is related to the average pore radius of the gas diffusion layer, $r_{s}$, the temperature, $T$, and the molecular weight of oxygen, $M .{ }^{23)}$

$$
D_{k s}=97 r_{s}(T / M)^{0.5}
$$

Based on the above eqns. (2) and (3), the 
partial pressure difference of oxygen across the gas diffusion layer can be predicted for a given flux of oxygen.

\subsubsection{Oxygen diffusion in gas channels of} reaction layer

Similarly to that mentioned above, oxygen diffusion in the gas channels of the reaction layer obeys the Knudsen diffusion. The relation of the partial pressure of oxygen, $P_{2}$, and the flux of oxygen, Jo2, is expressed by

$$
\frac{d P_{O_{2}}}{d z}=-\frac{R T J_{o_{2}}}{D_{k t} \varepsilon_{1} / \tau_{t}}
$$

where $a$ and $\tau$ are the porosity and tortuosity of the gas channels of the reaction layer. The Knudsen diffusion coefficient of oxygen in the gas channel of the reaction layer, $D_{\mathrm{kt}}$, is related to the average pore radius of the gas channels of the reaction layer, $n$, by

$$
D_{k t}=97 r_{t}(T / M)^{0.5} \text {. }
$$

\subsubsection{Diffusion of oxygen across thin-film,} and diffusion and reduction of oxygen in flooded-agglomerates

As oxygen enters the gas channels of the reaction layer, some of oxygen reacts along the way. The flux of oxygen diffusion past any point in the reaction layer along the axial direction, $z$, decreases in proportion to the reaction rate of oxygen per unit volume of electrode, ro2, at that point.

$$
\frac{d J_{O_{2}}}{d z}=-r_{O_{2}}
$$

Before oxygen enters the agglomerates of the reaction layer, it must first dissolve in the outer surface of the thin-film covering the agglomerates and diffuse across the film. According to the assumption (7), the dissolution of oxygen into the outermost layer of the thin-film can be taken to be in equilibrium with the partial pressure of oxygen, $P_{02}$, in the gas channels at that point and the concentration of oxygen in the outermost layer of the thin-film is $\mathrm{Po}_{2} / \mathrm{H}$, where $H$ is the Henry's constant of oxygen dissolution. The reaction rate of oxygen in the agglomerates of the reaction layer is equal to the diffusion flux of dissolved oxygen across the thin-film, which can be described approximately by the Fick's law based on the assumption (8).

$$
r_{O_{2}}=S_{t f} D_{L} \frac{P_{O_{2}}(z) / H-C_{O_{2}}\left(r_{a g}, z\right)}{\delta_{t h}}
$$

Where $D_{\mathrm{L}}$ is the diffusion coefficient of oxygen in electrolyte, $\delta_{t h}$ is the thickness of the thinfilm, $S_{t f}$ is the area of the film per unit volume of the reaction layer, $C_{2}\left(r_{a g}, z\right)$ is the concentration of oxygen at the interface of the thin-film and the flooded-agglomerate, and $r_{\text {ag }}$ is the radius of the agglomerate.

After diffused across the film, oxygen further diffuses and reacts in the porous flooded-agglomerates. The reduction rate of oxygen in porous electrodes has been found to be first order in the concentration of oxygen from the experimental investigation.4) According to assumption (9), the reaction rate of oxygen in the flooded agglomerates can be expressed by multiplying the rate constant, $k_{e}$, for the reaction, by the concentration of oxygen at the interface of the thin-film on floodedagglomerates, $\mathrm{Co}_{2}\left(r_{\mathrm{ag}}, z\right)$, by an effectiveness factor, $\eta_{\mathrm{eff}}$, and by the volume fraction of the agglomerates in the reaction layer of the electrode, $\chi_{\text {ag }}{ }^{42,43)}$

$$
r_{\mathrm{O}_{2}}=\chi_{a g} \eta_{a f f} k_{e} C_{\mathrm{O}_{2}}\left(r_{a g}, z\right)
$$

$\eta_{\text {off }}$ depends on the relative rates of diffusion and reaction, and is related to the Thiele modules, $\gamma$.

$$
\eta_{\text {aff }}=\frac{1}{\gamma} \tanh \gamma
$$

Where

$$
\gamma=L \sqrt{\frac{k_{a}}{D_{L, e f f}}}
$$

$L$ is the characteristic length of agglomerates. Since the agglomerate is assumed to be cylindrical, $L=r_{\mathrm{ag}} / 2$. $D_{\mathrm{L}, \mathrm{eff}}$ is the effective diffusion coefficient of dissolved oxygen in the flooded-agglomerate and is given by the 
diffusion coefficient of oxygen in electrolyte $D_{\mathrm{L}}$, corrected with the porosity, $\varepsilon_{\mathrm{n}}$, and the tortuosity, $\tau_{n}$ of the pores in the agglomerates of the reaction layer.

$$
D_{L, a f f}=D_{L} \varepsilon_{n} / \tau_{n}
$$

By eliminating $\mathrm{CO}_{\mathrm{O}}\left(\mathrm{rag}_{\mathrm{ag}}\right.$ z) from eqns. (7) and (8) and combining with eqn. (6), the gradient of $J_{02}$ at the axial direction, $z$, can be expressed in the reciprocal of the sum of the reciprocals of terms related to the diffusion in the film, and the diffusion and reaction in the agglomerates, since the diffusion of oxygen across the film and the diffusion and reaction of oxygen in the agglomerates occur in series.

$$
\frac{d J_{O_{2}}}{d z}=-\frac{P_{O_{2}}(z) / H}{\delta_{t 1} /\left(S_{v} D_{L}\right)+1 /\left(\chi_{a s} \eta_{e D} k_{u}\right)}
$$

\subsubsection{Reaction rate constant}

Since reaction of oxygen in the agglomerates is an electrochemical reduction reaction, the transfer current density per unit area of gas-electrolyte interface, itf, can be expressed by a Tafel equation. ${ }^{27)}$

$$
i_{\text {r }}=A C_{O_{2}}\left(r_{a g}, z\right) \exp _{10}\left[\left(E_{a}(z)-E_{l}(z)\right) / T_{s}\right]
$$

Where $A$ is a constant, $T \mathrm{~s}$ is the Tafel slope, and $\mathrm{CO}_{2}$ is the concentration of oxygen dissolved in the electrolyte, $E_{\theta}$ and $E$ are the electrical potential of the catalyst and carbon materials and that of the electrolyte, respectively. The constant $A$ is related to the exchange current density and can be estimated experimentally. For the reduction rate of oxygen, we have

$$
r_{o_{2}}=i_{v} S_{v} /(4 F)
$$

Combining eqn. (8) with eqns. (13) and (14), the reaction rate constant, $k_{\theta}$, can be expressed in the following equation.

$$
\begin{aligned}
k_{a} & =i_{v f} S_{v} /\left(4 F \chi_{a g} \eta_{a f f} C_{O_{2}}\left(r_{a g}, z\right)\right) \\
& =\frac{S_{v f} A}{4 F \chi_{a g} \eta_{a f}} \exp _{10}\left[\left(E_{d}(z)-E_{l}(z)\right) / T_{s}\right]
\end{aligned}
$$

\subsubsection{Electronic and ionic conduction.}

Ohm's law governs the current of electrons, $i_{\theta}$, through the catalyst and carbon materials of the flooded-agglomerates,

$$
\frac{d E_{\theta}}{d z}=-\frac{i_{e}(z)}{\kappa_{c}}
$$

and the current of total ions, $\dot{h}$, in the electrolyte of the flooded-agglomerates,

$$
\frac{d E_{i}}{d z}=-\frac{i_{i}(z)}{\kappa_{i}}
$$

where $\kappa_{c}$ is the electronic conductivity of the catalyst and carbon materials. The effective conductivity of the electrolyte in the pores of

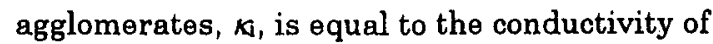
the free electrolyte, $\kappa_{1}, 0$, times the volume fraction of agglomerates, $\chi_{\mathrm{ag}}$, times the porosity of the pores in agglomerates, $\sigma_{h}$, and times the reciprocal tortuosity of the pores of agglomerates, $\tau_{\mathbf{n}}$.

$$
\kappa_{i}=\kappa_{i, 0} \chi_{a g} \varepsilon_{n} / \tau_{n}
$$

The flux of oxygen flowing through the plane at $z$ is consumed in producing the electronic current density at $z$, flowing in the opposite direction.

$$
i_{\theta}(z)=-4 F J_{O_{2}}(z)
$$

The ionic current density $i_{i}(z)$ is equal to the difference between the total electronic current density through the plane at $z=0$ and the electronic current density at $z$.

$$
i_{i}(z)=i_{\theta}(0)-i_{\theta}(z)
$$

$\Delta E(z)$ is defined as the difference between the potentials in the catalyst and carbon materials and that in the electrolyte.

$$
\Delta E(z)=E_{e}(z)-E_{i}(z)
$$

Therefore, differentiating eqn. (21) and combining it with eqns. (16) and (17), yield

$$
\frac{d \Delta E}{d z}=-\frac{i_{\theta}(0)}{\kappa_{j}}+4 F\left(\frac{1}{\kappa_{c}}+\frac{1}{\kappa_{i}}\right) J_{O_{2}}(z) \text {. }
$$




\subsubsection{Numerical calculation}

For a given operating current density, $i$, the flux of oxygen through the gas-diffusion layer, $J_{02}$, is obtained from the relation below,

$$
J_{O_{2}}=-i /(4 F) .
$$

The partial oxygen pressure, $P_{02,0}$, at the boundary-face of the gas diffusion layer and the reaction layer can be calculated by integrating eqn. (2),

$$
P_{o_{2}, 0}=P_{o_{2}}-\frac{R T z^{\prime} J_{O_{2}}}{D_{k s} \varepsilon_{v} / \tau_{s}}
$$

where $P_{02}$ is the partial pressure of oxygen at the gas-supply chamber, and $z$ is the thickness of the gas-diffusion layer.

The performance of the reaction layer can be characterized by solving the three simultaneous eqns. (4), (12), and (22). For a given operating current density, $i$, the value of $\Delta E(0)$ must be varied by trial and error to give a solution of the equations for which the oxygen flux through the interface of the catalyst reaction layer and the electrolyte bulk, $J_{2}\left(z_{1}\right)$, is equal to zero. The results obtained are the axial distributions of $J_{\mathrm{o}_{2}}, \mathrm{P}_{2}$ and $\Delta E$ over all reaction layer from $z=0$ to $z=z_{1}$, where $z_{1}$ is the thickness of the reaction layer, $i_{\theta}$ and $j$ can be obtained from Jo2 using eqns. (19) and (20), and $E_{\theta}$ and $E_{4}$ can be obtained from either eqns. (16) or eqn. (17) together with eqn. (21). Since $E_{\theta}$ and $E_{1}$ are not independently observable, the boundary conditions of $E_{1}$ is arbitrary and $E_{1}(0)$ is conveniently chosen as zero.

It is noted that, for a given value of $\Delta E(z)$, estimation of $k_{\theta}, \eta_{\theta \in f}$ and $\gamma$ can be done from eqns. (9), (10) and (15) by trial and error. At first, the initial value of $k_{\theta}$ can be obtained from eqn. (15) by assuming $\eta_{\text {eff }}=1.0$, where activation is the only source of voltage loss. Then introducing the above value of $k_{\theta}$ into eqn. (11) yields $\gamma$ and introducing $\gamma$ into eqn. (10) yields $\eta_{\text {erf. }}$ The value of $k_{e}$ can be modified again with eqn. (15) and this cyclic computation will be repeated until all of the three values of $k_{\theta}, \eta_{\text {erf }}$ and $\gamma$ approach to constant values, respectively.
The potential difference, $E_{1}\left(z_{1}\right)-E_{\theta}(0)$, between the potential of the electrolyte solution at the interface of the reaction layer and bulk electrolyte, $E_{1}\left(z_{1}\right)$, and the potential of solid phase (catalyst and carbon materials) at the boundary-face of the reaction layer and gas-diffusion layer, $E_{\theta}(0)$, can be measured experimentally. Thus we give the definition of overpotential, $\eta$, as follows.

$$
\eta=E_{i}\left(z_{1}\right)-E_{a}(0)-\Delta E_{\text {standard }}
$$

Here $\Delta E_{\text {standard }}$ is the standard potential of the reaction of the direct 4 electrons reduction of oxygen expressed by eqn.(1) and equal to $0.401 \mathrm{~V}$. In the present study, we focus on the calculation of overpotential as a function of current density under various conditions using a FORTRAN 77 program.

\section{PARAMETER ESTIMATION}

The base-case conditions of the structural parameters of oxygen diffusion electrodes are obtained from ref. 7. For the reaction layer, the thickness and porosity are $1.0 \times 10^{4} \mathrm{~m}$ and 0.30 , respectively; and the radii of the floodedagglomerates and the gas channels are $5.0 \times$ $10^{.6} \mathrm{~m}$ and $5.0 \times 10^{.8} \mathrm{~m}$, respectively. The volume fraction of the flooded-agglomerates is 0.50 and the thickness of the thin film on flooded-agglomerates is $5.0 \times 10^{-8} \mathrm{~m}$. For the gas-diffusion layer, the thickness, porosity and average pore radius are $5.0 \times 10^{-4} \mathrm{~m}, 0.50$ and $2.5 \times 10^{-8} \mathrm{~m}$, respectively. According to Kunz et al. ${ }^{26)}$ and $\mathrm{Li}$ et al., 38) the porosity of the gas channels of the reaction layer, $\approx$, and the specific area of gas-electrolyte interface, $S_{f}$, are expressed by the following equations, respectively.

$$
\begin{aligned}
& \varepsilon_{\mathrm{t}}=\varepsilon_{\mathrm{n}} \times\left(1.0-\chi_{\mathrm{ag}}\right), \\
& S_{\mathrm{ff}}=2.0 \times \varepsilon_{\mathrm{n}} \times \chi_{\mathrm{ag}} \times\left(1.0-\chi_{\mathrm{ag}}\right) / r_{\mathrm{ag}}
\end{aligned}
$$

In addition, the tortuosity of the gas-diffusion layer and gas-channels of the reaction layer is assumed to take the same value of 3.0. The properties of the electrodes mentioned above are used as the base-case conditions for the model calculation and listed in Table 1.

Pure oxygen and the catholyte of $30 \%(w t$. 
Table 1 The parameters of the base-case conditions.

\begin{tabular}{|c|c|}
\hline Quantity & $\begin{array}{l}\text { Value for base. } \\
\text { case condition }\end{array}$ \\
\hline $\begin{array}{l}\text { gas diffusion layer } \\
\text { thickness, } z^{\prime} \\
\text { porosity, } \\
\text { averaged pore radius, } n \\
\text { tortuosity, } \bar{n}\end{array}$ & $\begin{array}{l}6.0 \times 10^{-4} \mathrm{~m} \\
0.60 \\
2.5 \times 10^{-8} \mathrm{~m} \\
3.0 \\
\end{array}$ \\
\hline $\begin{array}{l}\text { reaction layer } \\
\text { thickness, } z_{1} \\
\text { porosity, on } \\
\text { radius of gas channel, } n \\
\text { porosity of gas channel, a } \\
\text { tortuosity of gas channel, } n \\
\text { radius of nooded-agglomerate, raz } \\
\text { volume fraction of flooded- } \\
\text { agglomerate, } \chi_{a z} \\
\text { thickness of thin film, } \delta_{t h} \\
\text { area of gas-liquid interface, } S\end{array}$ & $\begin{array}{l}1.0 \times 10^{-4} \mathrm{~m} \\
0.30 \\
6.0 \times 10^{-0} \mathrm{~m} \\
\delta_{\mathrm{n}} \times\left(1.0-\chi_{\mathrm{ad}}\right) \\
3.0 \\
6.0 \times 10^{-6} \mathrm{~m} \\
0.60 \\
5.0 \times 10^{-8} \mathrm{~m} \\
2.0 \times \sigma_{\mathrm{n}} \times x_{\mathrm{ag}} \times \\
\left(1.0-x_{\mathrm{ag}}\right) / r_{\mathrm{ag}} \\
\end{array}$ \\
\hline $\begin{array}{l}\text { electrochemical reaction } \\
\text { constant } A \\
\text { Tafel slope, } T_{\mathrm{s}}\end{array}$ & $\begin{array}{l}1.0 \times 10^{-3} \\
\mathrm{~A} \mathrm{~m} \mathrm{~mol} \cdot 1 \\
75 \mathrm{mV}\end{array}$ \\
\hline $\begin{array}{l}\text { operating condition } \\
\text { partial pressure of oxygen, } P_{2} \\
\text { temperature, } T\end{array}$ & $\begin{array}{l}1.0 \mathrm{~atm} \\
363 \mathrm{~K}\end{array}$ \\
\hline
\end{tabular}

$\mathrm{NaOH}$ solution are employed. The base-case conditions of operating temperature and partial pressure of oxygen in gas supply chamber are $363 \mathrm{~K}$ and $1.0 \mathrm{~atm}$, respectively.

Based on the data from Ogumi et al, ${ }^{44)}$ Bernardi and Verbrugge ${ }^{45}$ ) proposed the following equations to represent the Henry's constant of the oxygen dissolution and the diffusion coefficient of dissolved oxygen in $\mathrm{KOH}$ solution.

$$
\begin{aligned}
& H=1.33 \times 10^{6} \exp (-666 / T) \\
& D_{L . O_{2}}=3.1 \times 10^{-3} \exp (-2768 / T)
\end{aligned}
$$

Here, Henry's constant is in a unit of atm $\mathrm{cm}^{3}$ $\mathrm{mol}^{-1}$, the diffusion coefficient is in $\mathrm{cm}^{2} \mathrm{~s}^{-1}$ and $T$ is in Kelvin. It is assumed that the above equations are also approximately applicable for $\mathrm{NaOH}$ solution.

Ionic conductivity of oxygen diffusion electrode is estimated to be $1.0 \mathrm{~S}^{\cdot 1} \mathrm{~m}^{\cdot 1}$ from ref. 46 and electronic conductivity of free electrolyte solution, $\kappa_{0} 0$, is calculated from eqn. (30). 41,46$)$

$$
\kappa_{i 0}=\frac{F^{2}}{R T} \sum_{i} z_{1}^{2} D_{i} c_{i}
$$

Where, $F$ is the Faraday constant, and $R$ is molar gas constant. $z_{1} a$ and $D_{1}$ are the charge, the concentration and diffusion coefficient of the ions of species $i$ in the electrolyte.

From the experimental investigation, the Tafel slope of the oxygen diffusion electrode for an alkaline cell system was measured to take the values from 60 to $120 \mathrm{mV}$; we have assumed it to be $75 \mathrm{mV}$ for the base-case conditions. The constant $A$ has been determined so as to result in a good agreement between the simulated result and the experimentally-predicted ideal overpotential of $0.2-0.5 \mathrm{~V}$ at $300 \mathrm{~A} \mathrm{~m}^{2}{ }^{47)}$ Eventually the employed $1.0 \times 10^{-3} \mathrm{~A} \mathrm{~m} \mathrm{~mol}^{-1}$ results in $c 8.0 .3$ $V$. The change in $A$ by one order results in the change of overpotential of $75 \mathrm{mV}$ corresponding to the Tafel slope value. The qualitative discussions of the parameter effects are not affected by the change in the $A$ value.

\section{RESULTS AND DISCUSSION}

Our attention in the model calculation will be focused on the dependence of overpotential voltage on the structural parameters of oxygen diffusion electrodes and the operating conditions (such as temperature and oxygen partial pressure). The structural parameters of oxygen diffusion electrodes investigated and their base-case conditions are shown in Table 1.

Figure 2 shows the semi-logarithmic plot between the overpotential and the current density under three different values of porosity of the reaction layer. At low current densities (< $100 \mathrm{~A} \mathrm{~m}^{-2}$ ), the overpotential increases linearly with increasing current density due to activation of oxygen reduction reaction; at higher current densities, the overpotential increases remarkably and the current densities approach to the maximum values, they are called the limited current density. The larger the porosity of the reaction layer is, the higher the current densities at the same overpotential are (or in other words, the lower the 


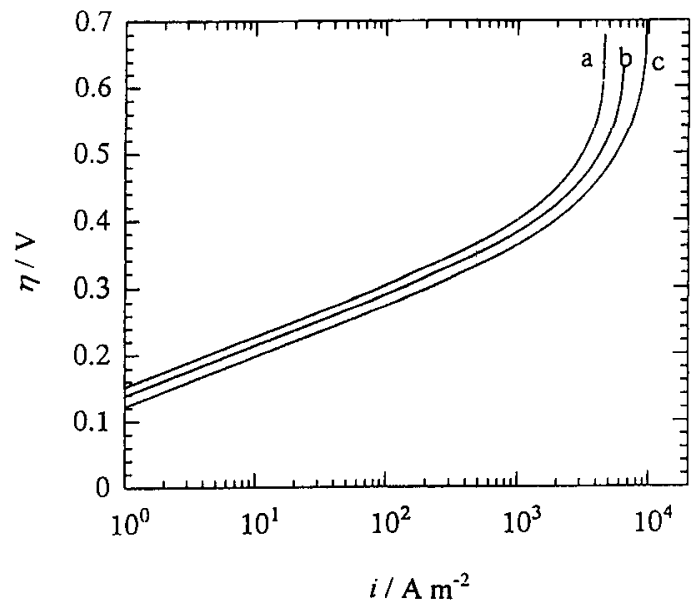

Fig. 2 Overpotential, $\eta$, vs. current density, $i$, as a function of the porosity of the reaction layer with the values: a) 0.2 , b) 0.3 , and c) 0.5 .

overpotential at the same current density is) This is because with the increase of the porosity of the reaction layer, the area of the thin-film on the flooded-agglomerates becomes larger. The porosity of the reaction layer of the electrode investigated $w$ as designed to be about 0.28 .7) A larger value of the porosity of the reaction layer is desirable based on the calculation result shown in Fig. 2. However, it may cause the gas percolation into the catholyte chamber easily as discussed in the preceding paper. ${ }^{8)}$ Therefore, the suitable porosity of the reaction layer must be considered from the two viewpoints mentioned above.

Figure 3 shows the curves of the overpotential vs. the current density by varying the thickness of the thin-film on the agglomerates in the reaction layer. At lower current densities, the effects of the thickness of the thin-film are negligible; and at higher current densities, the thinner the thickness of the thin-film is, the lower the overpotential is. The maximum current density is limited by the diffusion of dissolved oxygen through the thinfilms and decreases with the increase of the thickness of the thin-film. How to control the thickness of the thin-film is not yet known,

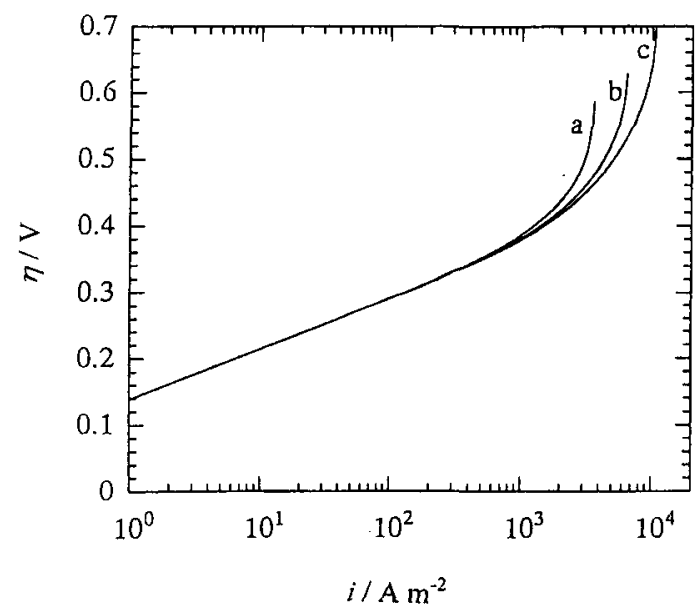

Fig. 3 Overpotential, $\eta$, vs. current density, $i$, as a function of the thickness of the thin-film on the flooded-agglomerates in the reaction layer with the values: a) 2.0 x $10^{-7} \mathrm{~m}$, b) $1.0 \times 10^{-7} \mathrm{~m}$, and c) $5.0 \times 10^{-8} \mathrm{~m}$.

which is, however, supposed to be related to the materials and the preparation method of the electrodes.

The effects of the radius of the floodedagglomerates in the reaction layer, $r_{a g}$, on the curves of the overpotential vs. the current density are shown in Fig. 4. With the radius of

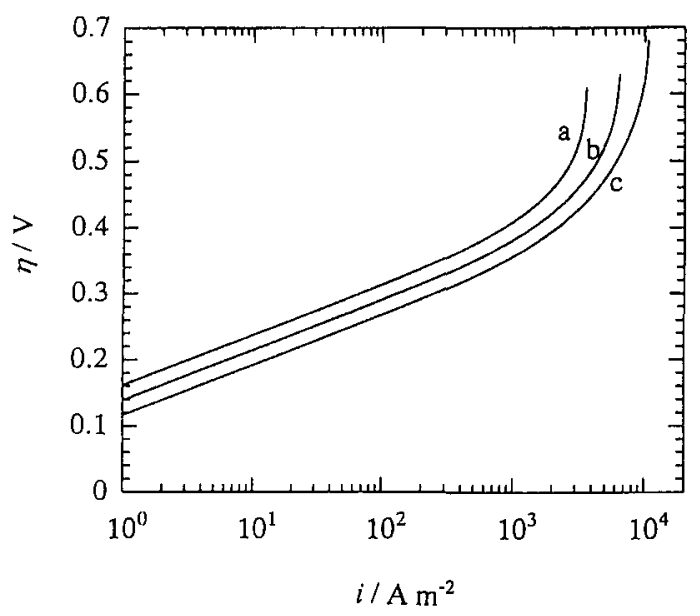

Fig. 4 Overpotential, $\eta$, vs. current density, $i$, as a function of the radius of the flooded-agglomerates in the reaction layer with the values: a) $2.0 \times 10^{-5} \mathrm{~m}$, b) $1.0 \times 10^{.5}$ $\mathrm{m}$, and c) $5.0 \times 10^{-6} \mathrm{~m}$. 
the flooded-agglomerates becoming smaller, the area of the thin-film on the floodedagglomerates increases and consequently the current density gets larger at the $\operatorname{sam} \theta$ overpotential. Although the radius of the flooded-agglomerates can not be measured directly, it can be estimated from the spectrum of the pores and the volume of intruded electrolyte in the reaction layer. ${ }^{27)}$ The spectrum of the pores can be measured by a mercury porosimeter; and the volume of intruded electrolyte can be calculated from the difference between the weight of the electrode before testing and that of the same electrode after tested. ${ }^{46)}$ The radius of the floodedagglomerates is related to the preparation method: the size of carbon particles, the ratio of hydrophilic carbon to hydrophobic carbon, the content of PTFE used for the preparation of the reaction layer of the electrode.

Figure 5 shows the curves of the overpotential vs. the current density by varying the volume fraction of the floodedagglomerates in the reaction layer, $\chi_{\text {ag }}$ from 0.1 to 0.9. The effects of $\chi_{\text {ag }}$ can be divided into two regions. For the region where $\chi_{\text {ag }}$ is smaller than 0.5 , the overpotential at the same current

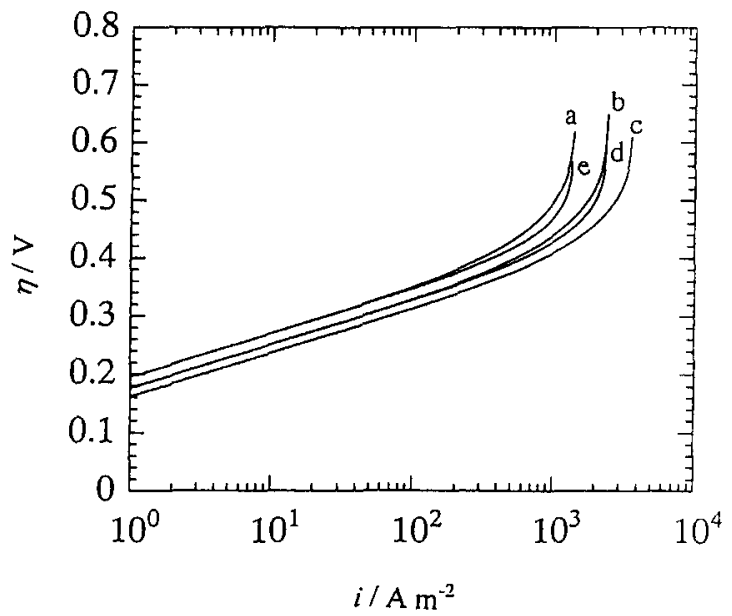

Fig. 5 Overpotential, $\eta$, vs. current density, $i$, as a function of the volume fraction of the thin-film and the floodedagglomerates in the reaction layer with the values: a) 0.1, b) 0.2 , c) 0.5 , d) 0.8 , and $e$ ) 0.9 . density decreases with the growth of $\chi_{\text {ag }}$ from 0.1 to 0.5 ; while, for the region where $\chi$ ag is larger than 0.5 , the overpotential at the same current density increases with the growth of $\chi_{\text {ag }}$ from 0.5 to 0.9 . At the crossing point of the two regions, $\chi_{\mathrm{ag}}=0.5$, there exists the curve of minimum overpotential among the all curves of the overpotential vs. the current density, since here the specific area of the gas-electrolyte reaches to a maximum value as is derived from eqn. (27).

It is noted that the minimum value of overpotential appearing at $\chi_{\mathrm{ag}}=0.5$ is only applicable for the electrodes whose specific area of the gas-electrolyte obeys the relation of eqn. (27). For most electrodes, the minimum values of overpotential need not appear at $\chi_{\mathrm{ag}}=$ 0.5 , although they appear certainly for the case when the specific area of the gas-electrolyte reaches to a maximum value. Assuming that the reaction layer has uniform porosity everywhere, $\chi_{\text {ag }}$ is considered to be the approximate ratio of the volume of intruded electrolyte to the total free volume of the reaction layer. Since the volume of intruded electrolyte depends on the operation conditions (i.e. the concentration of catholyte, the temperature and pressures of the electrolyzer) and also doesn't hold constant after a long time

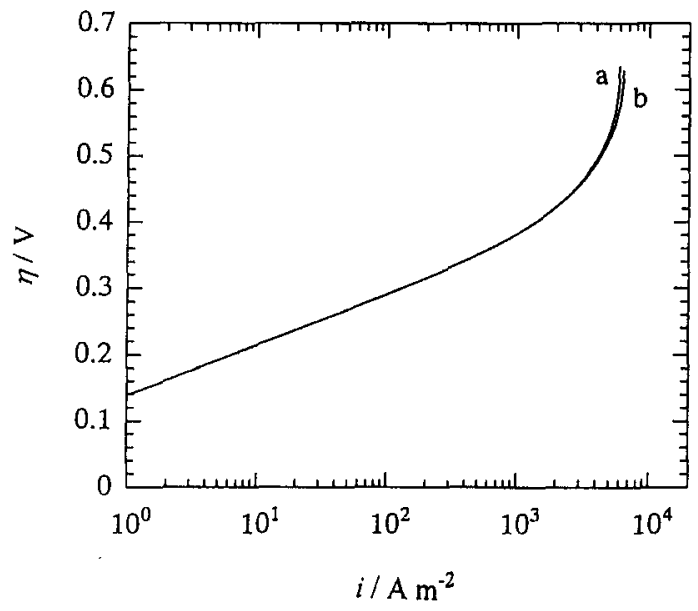

Fig. 6 Overpotential, $\eta$, vs. current density, $i$, as a function of the radius of the gas channels in the reaction layer with the values: a) $1.0 \times 10^{-8} \mathrm{~m}$, and b) $5.0 \times 10^{-8} \mathrm{~m}$. 
operation, the curves of the overpotential vs. the current density will change accordingly.

As is shown in Fig. 6, there are very little change of the curves of the overpotential vs. the current density as a function of the radius of the gas channels in the reaction layer from 50 $\mathrm{nm}$ to $10 \mathrm{~nm}$. The results indicate that the adopted size of the gas channels is large enough for the oxygen to go through it and

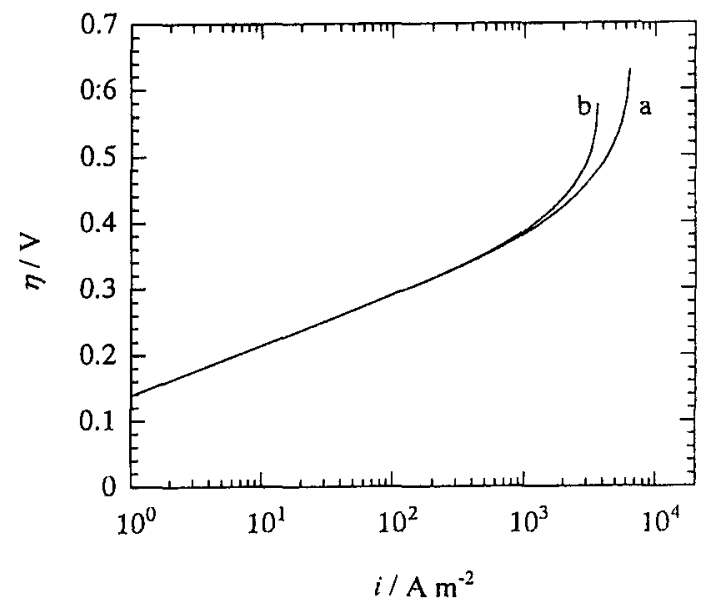

Fig. 7 Overpotential, $\eta$, vs. current density, $i$, as a function of the average pore radius of the gas-diffusion layer with the values: a) $2.5 \times 10^{-8} \mathrm{~m}$, and b) $5.0 \times 10^{-9} \mathrm{~m}$.

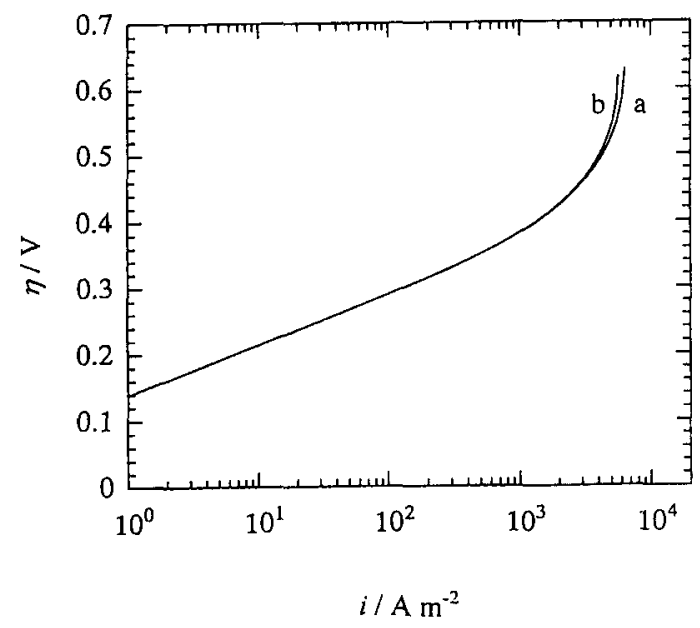

Fig. 8 Overpotential, $\eta$, vs. current density, $i$, as a function of the porosity of the gas-diffusion layer with the values: a) 0.5 , and b) 0.3 . reach the gas-liquid interface, or in other words, the resistance of the Knudsen diffusion of the oxygen through the gas channels is very small.

Figures 7 and 8 show the effects of the average pore radius and the porosity of the gas diffusion layer on the curves of the overpotential vs. the current density. If the average pore radius becomes much smaller (from $25 \mathrm{~nm}$ to $5 \mathrm{~nm}$ ), the resistance of Knudsen diffusion of the oxygen through the gas diffusion layer becomes larger and the oxygen partial pressure at the boundary-face of the reaction layer and the gas diffusion layer becomes smaller.

\section{CONCLUDING REMARKS}

The TFFA model assumes that the oxygen diffusion electrode made of a gas-diffusion layer and a reaction layer possesses a doublescales of porous structure in the reaction layer, and it has given a detailed description of all of the processes that include diffusion of oxygen in the gas-diffusion layer and the gas-channels of the reaction layer, dissolution and diffusion of oxygen in the thin-film of the reaction layer, diffusion and reaction of oxygen in the floodedagglomerates of the reaction layer and the ionic and electronic conduction.

The electrode performance is expressed by the curve of the overpotential vs. the current density and its dependence on the structural parameters has been investigated. Among them, much attention must be paid to the porosity of the reaction layer, the thickness of the thin-film on the flooded-agglomerate, the radius of the flooded-agglomerate, the volume fraction of the flooded-agglomerates in the reaction layer. The radius of the gas channels in the reaction layer and the porosity and average pore radius of the gas-diffusion layer seem to be less important for the performance of the electrode. The above suggestions are mainly derived from the fact that the Knudsen diffusion of oxygen is much faster than the diffusion and reaction of dissolved oxygen in the thin-film and flooded-agglomerates of the reaction layer of the electrode. 
A preparation method to keep most of structural parameters, such as the volume fraction of the thin-film and the floodedagglomerates, in adequate values is very desirable, though the relationship between the preparation method of the electrode and the structural parameters mentioned in this paper is still uncertain. The scale-up of the oxygen diffusion electrode for a chlorine-alkali electrolysis system might make it more difficult to keep the structure parameters in most suitable ranges irrespective of variation of the depth of the catholyte, and the difference between the catholyte chamber and the gassupply chamber of the electrolyzer for a long time operation.

This work is partly supported by a Grantin-Aid for Scientific Research (B) from the Ministry of Education, Science, Sports and Culture of Japan (No. 08555187), which is greatly appreciated. We are also very grateful to Japan Soda Industry Association and the Society of Chemical Engineers, Japan for giving us the opportunity to be involved in this work. The discussion and comments given by Mr. Y. Aikawa (Mitsui Toatsu Chemicals, Inc.), Mr. K. Saiki (Kaneka Corp.), Mr. A. Sakata (Toagosei Co., LTD.), Prof. M. Sudoh (Shizuoka Univ.), Prof. H. Nagamoto (Kogakuin Univ.), and Prof. H. Kameyama (Tokyo Univ. of Agric. $\&$ Tech.) have been very valuable. We refer great thanks to them.

\section{REFERENCES}

1) S. Motoo, M. Watanabe and N. Furuya, $J$. Electroanal. Chem., 160, 351(1984).

2) M. Watanabe, M. Tomikawa and S. Motoo, J. Electroanal. Chem., 182, 193 (1985).

3) M. Watanabe, M. Tomikawa and S. Motoo, J. Electroanal. Chem., 195, 81(1985).

4) T. Kenjo and K. Kaw atsu, Electrochim. Acta, 30, 229 (1985).

5) K. Horita, G. Kano and Y. Nomura, Denki Kagaku, 57, 582 (1989).

6) Y. Sato, M. Sato and M. Sakaguchi, Denki Kagaku, 57, 1000 (1989).

7) The Society of Chemical Engineers of
Japan, Annual report on the investigation of the scale-up of gas diffusion electrodes (1995).

8) X. L. Wang and S. Koda, Denki Kagaku, in press. (1997).

9) L. G. Austin, M. Ariet, R. D. Walker, G. B. Wood and R. H. Comyn, Ind. Eng. Chem. Fundam., 4, 321 (1965).

10) R. Brown and J. A. Rockett, $J$. Electrochem. Soc., 113, 865 (1966).

11) S. Srinivasan, H. D. Hurwitz and J. O' M. Bockris, J. Chem. Phys., 46, 3108 (1967).

12) J. O' M. Bockris and B. D. Cahan, $J$. Chem. Phys., 50, 1307 (1969).

13) G. P. Sakellaropoulos and S. H. Langer, AIChE. J., 24, 1115 (1978).

14) K. Y. Chan, G. S. Efthymiou and J. F. Cocchetto, Electrochim. Acta, 32, 1227 (1987).

15) F. G. Will, J. Electrochem. Soc., 110, 152 (1963).

16) R. P. Iczkowski, J. Electrochem. Soc., 111 , 1078 (1964).

17) J. A. Rockett and R. Brown, $J$. Electrochem. Soc., 113, 207 (1966).

18) S. Srinivasan and H. D. Hurwitz, Electrochim. Acta, 12, 495 (1967).

19) E. A. Grens II, Ind. Eng. Chem. Fundam., 5, 543 (1966).

20) J. Giner and C. Hunter, J. Electrochem. Soc., 116, 1124 (1969).

$21)$ W. M. Vogel and J. T. Lundquist, Electochim. Acta, 17, 1735 (1972)

22) D. C. Chan and C. C. Wan, J. Power Sources, 50, 261 (1994).

23) M. B. Cutlip, Electrochim. Acta, 20, 767 (1975).

24) R. P. Iczkowski and M. B. Cutlip, J. Electrochem. Soc., 127, 1433 (1980).

$25)$ C. Y. Yuh and J. R. Selman, J. Electrochem. Soc., 131, 2062 (1984).

26) H. R. Kunz, L. J. Bregoli and S. T. Szymanski, J. Electrochem. Soc., 131, 2815 (1984).

27) P. Bjornbom, Electochim. Acta, 32, 115 (1987).

28) E. A. Ticianelli, S. Srinivasan, C. R. Derouin and A. Redondo, J. Electrochem. 
Soc., 135, 2209 (1988).

29) T. E. Springer and I. D. Raistrick, J. Electrochem. Soc., 136, 1594 (1989).

30) S. C. Yang and M. B. Cutlip, Electochim. Acta, 34, 703 (1989).

31) M. B. Cutlip, S. C. Yang and P. Stonehart, Electochim. Acta, 35, 869 (1990).

32) S. C. Yang, M. B. Cutlip and P. Stonehart, Electochim. Acta, 36, 547 (1991).

33) I. D. Raistrick, Electochim. Acta, 35, 1579 (1990).

34) H. Celiker, M. A. Al-Saleh, S. Gultekin and A. S. Al-Zakri, J. Electrochem. Soc., 138, 1671 (1991).

35) C. Y. Yuh and J. R. Selman, J. Electrochem. Soc., 139, 1373 (1992).

36) S. C. Yang and P. Bjornbom, Electrochim. Acta, 37, 1831 (1992).

37) J. Perez, A. A. Tanaka, E. R. Gonzalez and E. A. Ticianelli, J. Electrochem. Soc., 141, 431 (1994).

38) Q. Li, X. Gang, H. A. Hjuler, R. W. Berg and N. J. Bjerrum, J. Electrochem. Soc., 141,3114 (1994).

39) Y. W. Rho, S. Srinivasan and Y. T. Kho, J. Electrochem. Soc., 141, 2089 (1994).

40) E. A. Ticianelli, J. Electroanal. Chem. Soc., 387, 1 (1995).

41) M. C. Kimble and R. E. White, J. Electrochem. Soc., 138, 3370 (1991).

42) G. R. Youngquist, Ind. Eng. Chem., 62, 52 (1970).

43) G. F. Froment and K. B. Bischoff, "Chemical Reactor Analysis and Design", pp.157-181, 2nd-ed., John Wiley \& Sons, Inc. (1990).

44) Ogumi, Z., Z. Takehara and S. Yoshizawa, J. Electrochem. Soc., 131, 769 (1984).

45) D. M. Bernardi and M. W. Verbrugge, AIChE J., 37, 1153 (1991).

46) D. B. Zhou and H. V. Poorten, Electrochim. Acta, 40, 1819 (1995).

47) Y. Aikawa, Soda \& Chlorine, 45, 85 (1994). 